\title{
Adaptive Filtering and Prediction of the Southern Oscillation Index
}

\author{
Christian L. KePPEnNE ${ }^{1}$ and Michael GhiL ${ }^{2}$ \\ Climate Dynamics Center and Department of Atmospheric Sciences, University of California, Los Angeles
}

\begin{abstract}
Singular spectrum analysis (SSA), a variant of principal component analysis, is applied to a time series of the Southern Oscillation index (SOI). The analysis filters out variability unrelated to the Southern Oscillation and separates the high-frequency, 2- to 3-year variability, including the quasi-biennial oscillation, from the lower-frequency 4- to 6-year El Niño cycle. The maximum entropy method (MEM) is applied to forecasting the prefiltered SOI. Prediction based on MEM-associated autoregressive models has useful skill for 30-36 months. A 1993-1994 La Niña event is predicted based on data through February 1992.
\end{abstract}

\section{INTRODUCTION}

The atmospheric Southern Oscillation (SO) has been connected with the seasonally recurring, oceanic El Niño (EN) phenomenon [e.g., Schweigger, 1945] by Bjerknes's [1966] dynamic and thermodynamic considerations. The implications of this El Niño/Southern Oscillation (ENSO) coupled ocean-atmosphere oscillation for tropical and global climate have led to many studies in recent years [e.g., Deser and Wallace, 1987].

The dynamic understanding of the coupled tropical oceanatmosphere system has increased dramatically [Cane, 1986; Philander, 1990], leading to increased hopes for its dynamic prediction on interannual time scales [Sarachik, 1990]. Multivariate statistical prediction models of ENSO have been investigated by Graham et al. $[1987 a, b]$ and Barnett et al. [1988]. Their statistical models are based on extended empirical orthogonal functions (EEOFs) and canonical correlation analysis and exhibit valuable forecast skill at lead times of up to a year.

The above mentioned models rely on the analysis of sea surface temperature and surface wind stress fields that can serve as multivariate indicators of ENSO variability [e.g., Barnett and Preisendorfer, 1978]. The present study uses a time domain approach based on a univariate indicator of ENSO, rather than a space-time approach. Its goal is not to precisely forecast the spatial distribution of meteorological variables but rather to time the occurrence of EN and La Niña (LN) events, which are usually associated with recurring spatial patterns.

The use of univariate time series in the diagnosis and prediction of nonlinear dynamical systems with considerable complexity in time and space has a solid foundation in the Whitney [1936] embedding lemma and the Takens theorem [Mañe, 1981; Takens, 1981; Sauer et al., 1991]. Certain oversimplifications in the application of the univariate approach to climatic time series have been criticized with some justification [Grassberger, 1986; Procaccia, 1988; Ruelle, 1990]. However, the underlying idea of a time series from a

\footnotetext{
${ }^{1}$ Also at Jet Propulsion Laboratory, California Institute of Technology, Pasadena.

${ }^{2}$ Also at Institute of Geophysics and Planetary Physics, University of California, Los Angeles.

Copyright 1992 by the American Geophysical Union.

Paper number 92JD02219.

0148-0227/92/92JD-02219\$05.00
}

complex system being able to capture the evolution of its collective behavior is heuristically appealing and, when applied with due care, quite promising [Ghil et al., 1991]. In fact, this is precisely why the Southern Oscillation index (SOI) has attracted such attention, since the classical work of Walker and Bliss [1937] and up to the present [Fraedrich, 1988; Dickey et al., 1992], in describing the complex interannual variability of the coupled atmosphere-tropical ocean system.

Compositing of observations [Ghil and Mo, 1991a,b] or of model fields [Neelin et al., 1992] with respect to the phases of such a well-chosen time series provides an orderly sequence of snapshots of the system's past evolution. It can be combined at a later stage with suitably tested predictions of the same univariate index into the future, to yield eventually whole-field predictions. Thus univariate prediction is simply a first step in an approach that may inform and complement dynamical and multivariate statistical prediction [Vautard et al., 1992].

Univariate SOIs are generally computed from local time series of sea level pressure (SLP) or temperature data at two distinct locations along the equatorial belt, at which the variables under consideration tend to oscillate with mutually opposite phases [e.g., Walker and Bliss, 1937; Chu and Katz, 1985]. Our study relies on the analysis of such an SOI, but, unlike earlier attempts at univariate ENSO prediction [Chu and $\mathrm{Katz}, 1985$ ], our forecasts are based on a set of prefiltered SOI time series, rather than on the raw SOI itself.

The data and numerical procedures are presented in section 2 . In section 3 , singular spectrum analysis, a variant of principal component analysis (PCA) in the time domain, is applied to a SOI time series to isolate the temporal principal components (T-PCs) corresponding to ENSO activity from the remaining variability and noise. The linear predictability of the filtered SOI is examined in section 4 based on autoregressive models associated with prefiltered time series which isolate the variance corresponding to its four leading T-PCs. A summary and brief discussion of the results follow in section 5 .

\section{Data and Numerical Procedures}

\subsection{The Data}

The data consist of time series of monthly mean SLP at Tahiti and Darwin, Australia, from July 1941 to February 1992. The SOI time series is obtained here by first removing 


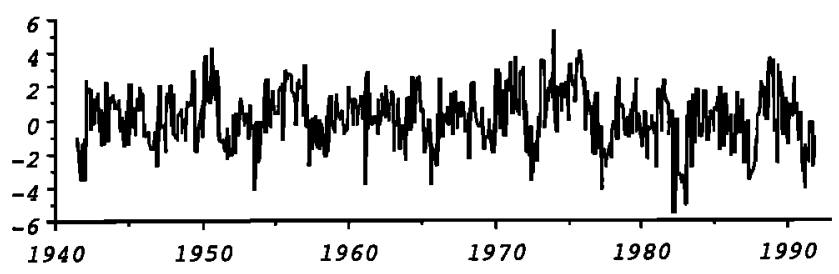

Fig. 1. Time series of the Tahiti-minus-Darwin SOI index from July 1941 to February 1992 . Tick marks on the abscissa, in this and subsequent figures, indicate January 15 of the year named.

the annual cycle (this is done by subtracting from either time series the mean SLP value at that location for the corresponding month), dividing the monthly anomalies so obtained by the corresponding standard deviation, and then taking the Darwin-minus-Tahiti difference [e.g., Trenberth and Shea, 1987]. The resulting time series is shown in Figure 1.

Continuous SLP records are available at Darwin since 1882 and at Tahiti since 1935; the continuous SLP time series at Darwin from January 1882 to December 1989 was analyzed and discussed by Keppenne and Ghil [1990]. Improvements in the quality of measurements since World War II and problems with the stationarity of the time series revealed by the earlier analysis have lead us to ignore the pre-1941 data in the present study.

\subsection{Singular Spectrum Analysis (SSA)}

SSA is the term used in a number of recent climate studies [e.g., Vautard and Ghil, 1989; Rasmusson et al., 1990; Ghil and Vautard, 1991] to refer to the univariate application of PCA [e.g., Preisendorfer, 1988] in the time domain. The method is also known as Karhunen-Loève (K-L) expansion [e.g., Pike et al., 1984] in digital signal processing. It was introduced into biological oceanography by Colebrook [1978], into nonlinear dynamics by Broomhead and King [1986], and into paleoclimatology by Fraedrich [1986]. Rasmusson et al. [1990] also used SSA to investigate the quasi-biennial component of ENSO.

SSA is algorithmically equivalent to the application of extended empirical orthogonal functions (EEOFs) [e.g., Weare and Nasstrom, 1982; Lau and Chan, 1986; Graham et $a l ., 1987 a, b]$ to a univariate time series but has special features and greater flexibility when applied to the analysis of phenomena with longer time scales and higher sampling rates [Mo and Ghil, 1992]. Vautard et al. [1992] provide an up-to-date review of SSA and of its applications to dataadaptive filtering and noise reduction. For brevity, we sketch here the method based on its relation to spatial empirical orthogonal function (EOF) analysis [e.g., Preisendorfer, 1988], which is of more common use in meteorology.

Spatial EOF (S-EOF) analysis proceeds by expanding the history of a discrete field $x_{i, j}$, where the indices $i$ and $j$ refer to the spatial and temporal directions respectively-1 $\leq i \leq$ $M, 1 \leq j \leq N$-into the sets of its eigenvectors (EOFs) and principal components (PCs). In SSA, the spatial direction is replaced by time lags, i.e., $x_{i, j}=x_{j+i}$, and $M$ becomes the number of lags. The algebraic formulation remains essentially the same but the T-PCs are shorter than the original time series by $M-1$ components. Vautard and Ghil [1989] refer to the EOFs and PCs of SSA as T-EOFs and T-PCs to distinguish them from their counterparts in S-EOF analysis.

The time scales of the dynamics addressed by SSA are bounded from below by the sampling interval, $\tau$, and from above by the window width, $\tau_{w}=M \tau$ [Vautard et al., 1992]. The choice of $M$ is a trade-off between the amount of information one wishes to retain and the degree of statistical significance that is required. Increasing $M$ enhances the former at the expense of the latter, and vice versa.

In contrast with standard spectral analysis in which the basis functions are given a priori (e.g., the sines and cosines of Fourier analysis), in SSA they are determined from the data themselves to form an orthogonal basis that is optimal in the statistical sense. Oscillatory modes can be identified as pairs of nearly equal eigenvalues, while their eigenfunctions (T-EOFs) and T-PCs have the same time scale of oscillation, as well as being nearly $90 \mathrm{deg}$ out of phase [Vautard and Ghil, 1989; Vautard et al., 1992]. Because of this property, the method is particularly helpful in isolating anharmonic oscillations with fluctuating amplitudes from noisy data.

The part of the time series' variability corresponding to a given oscillation can be isolated by restricting the K-L expansion to the T-EOFs and T-PCs that have been identified as corresponding to that oscillation [Ghil and Vautard, 1991; Vautard et al., 1992]. The reconstructed components (RCs) which carry the contributions of the individual T-EOFs and T-PCs to the variance of the data are time series of length $N$ (not $N-M+1$, like the T-PCs). The RCs are additive and their complete sum gives back the original time series.

The eigenvalue associated with a T-EOF gives the variance of the corresponding T-PC, while its square root is the associated singular value (SV). The SVs are standard deviations and give their name to the SSA method.

Individual T-PCs are not pure sine waves, but have a very limited harmonic content. Hence autoregressive (AR) models perform better in predicting the individual T-PCs than the time series itself. In section $\mathbf{4}$ an improved SOI forecast is obtained from the forecasts of its four leading RCs.

\subsection{Maximum Entropy: Spectral Estimation and Linear Prediction}

The main advantage of the maximum entropy method (MEM) [Yule, 1927; Walker, 1931; Burg, 1968] is its high spectral resolution, obtained by fitting relatively high-order AR models to the data. Its main drawback is the possible appearance of spurious peaks as the resolution, and hence the order, of the method is increased [Childers, 1978].

The basic assumption underlying MEM is that the time series can be modeled by an AR process. The optimal order of the AR process for a given time series is usually inferred from Akaike's information criterion (AIC) [Akaike, 1974]. However, the AIC often calls for a very high order if the data have not been prefiltered. SSA can be used to compute a data-adaptive prefilter by retaining only the leading, statistically significant T-PCs of a given time series [Ghil and Vautard, 1991; Vautard et al., 1992]. Removal of the noise by SSA permits the application of a low-order MEM, which achieves the same resolution as a much higher-order one, without the introduction of spurious peaks. This two-step procedure for spectral estimation is discussed in detail and applied to synthetic examples and to time series of atmospheric angular momentum by Penland et al. [1991]. 
(a)

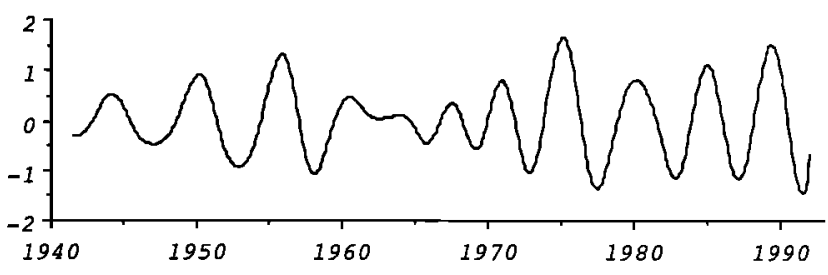

(b)

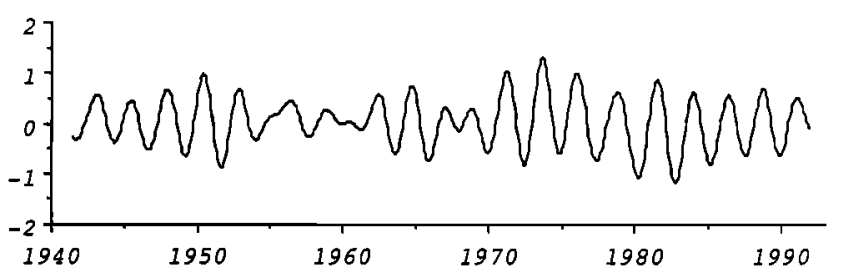

Fig. 2. Reconstructed time series obtained by combining the variance associated with two pairs of temporal principal components (T-PCs): (a) 1 and 2, the low-frequency (LF) ENSO component; (b) 3 and 4, the high-frequency (HF) ENSO component. The vertical scale in this and subsequent figures is in units of the standardized SOI of Figure 1.

Linear prediction (LP) using the AR coefficients provided by MEM is especially successful in extrapolating signals which are smooth and oscillatory, though not necessarily periodic [Press et al., 1988]. Its application to the individual T-PCs produced by an earlier SSA to forecast a prefiltered time series was introduced by Keppenne and Ghil [1990] and is discussed by Vautard et al. [1992]. In section 4, Burg's [1968] MEM algorithm is used to build AR predictors for the four leading $\mathrm{RCs}$ of the SOI data. This algorithm provides an efficient, recursive procedure to solve for the AR coefficients by exploiting the symmetric, Toeplitz-matrix structure of the autocovariance matrix associated with the time series [Press et al., 1988, pp. 447-466]. We prefer Burg's method to the traditional Yule-Walker approach because it does not assume any implied periodicity of the analyzed time series. The advantage of applying MEM to the RCs rather than to the original time series comes from their reduced frequency spectrum, in addition to their being relatively noise-free.

\section{SSA RESULTS}

SSA is applied to the SOI data with a window width of $\tau_{w}$ $=60$ months; here $\tau=1$ month and $M=60$. This width permits us to capture the low-frequency (LF) ENSO oscillations [cf. Rasmusson et al., 1990], while still providing a high degree of statistical significance. Two pairs of nearlyequal singular values (SVs), each capturing an oscillatory mode, are identified. These are SVs 1-2 and 3-4, which correspond to the LF component of ENSO and its highfrequency (HF) variability, including the quasi-biennial oscillation (QBO) [Rasmusson et al., 1990], respectively.

The LF and HF components of ENSO are isolated by summing the contributions of T-PCs 1 and 2 and T-PCs 3 and 4 respectively, using the reconstruction method justified rigorously by Vautard et al. [1992, equation (2.17)]. The resulting time series are shown in Figures $2 a$ and $2 b$. The total variance associated with ENSO combines the LF and $\mathrm{HF}$ components of Figures $2 a$ and $2 b$. This combined ENSO time series is shown in Figure 3 (solid) where it is compared with a 5-month running mean of the original SOI time series (dotted). The 5-month running mean is routinely used at the U.S. National Meteorological Center (NMC) for noise reduction and enhancement of the ENSO cycle; the correlation between the SSA-filtered and 5-month running mean SOI is 0.817 . The solid arrows indicate the minor warm episodes of 1941, 1946, 1951, 1953, 1957, 1965, 1969, 1977, and 1987, and the major ones of 1972, 1982, and 1992. The open arrows point to the cold events of 1950, 1956, 1971, 1974, 1976, and 1988. This labeling of cold and warm events is broadly accepted [e.g., Rasmusson and Carpenter, 1982; Deser and Wallace, 1987]. The additional smoothness of the SSA-filtered SOI is obtained without significant loss of resolution. This smoothness, which reflects that of the underlying T-PCs, is crucial to the success of the MEM forecasts of section 4 .

The eigenvectors associated with SVs 1-4, i.e., with ENSO variability, represent $21.7 \%$ of the total variance; that is, the solid curve in Figure 3 has $21.7 \%$ of the variance of the curve in Figure 1. The LF component associated with SVs 1 and 2 carries $11.4 \%$ of the variability (Figure $2 a$ ) and the remaining $10.3 \%$ are carried by the $\mathrm{HF}$ component associated with SVs 3 and 4 (Figure $2 b$ ). The HF and LF components of ENSO are thus of comparable magnitude, as noted by Rasmusson et al. [1990], based on surface wind data.

SVs 5-8 carry variability in the 1-2 year frequency band. The remaining SVs are close to or within the noise floor, as evidenced by a break in the slope of the singular spectrum [Vautard and Ghil, 1989] after SV 8. They correspond to the part of the variance that is unlikely to be explained by a deterministic model ( $64 \%$ of total variance).

\section{Predictability}

In this section we discuss how the predictability of the SOI can be improved by fitting an AR predictor to RCs 1-4. The idea is that the narrow harmonic content of these time series makes them more predictable by MEM than the original SOI time series. ENSO forecasts are obtained by summing the forecasts corresponding to the four prefiltered time series.

Figure $4 a$ illustrates the forecast skill of our method. It compares the last 10 years of the SSA-filtered SOI (solid) and 5-month running mean SOI (dotted), with the 36-month lead forecasts (crosses) obtained as follows. The last 10 years of data are removed from the unfiltered SOI time series, and SSA is applied to the remaining data. The

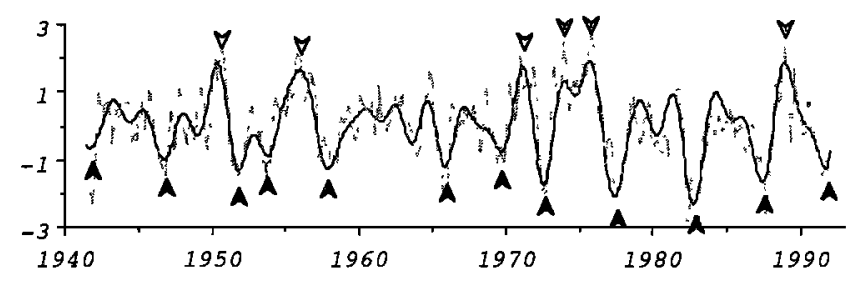

Fig. 3. Comparison of the SSA-filtered SOI computed from T-PCs and T-EOFs $1-4$ (solid) with a 5-month running mean of the conventional SOI (dotted). The open arrows point to cold (La Niña) events, the filled ones to warm (EI Niño) events. 

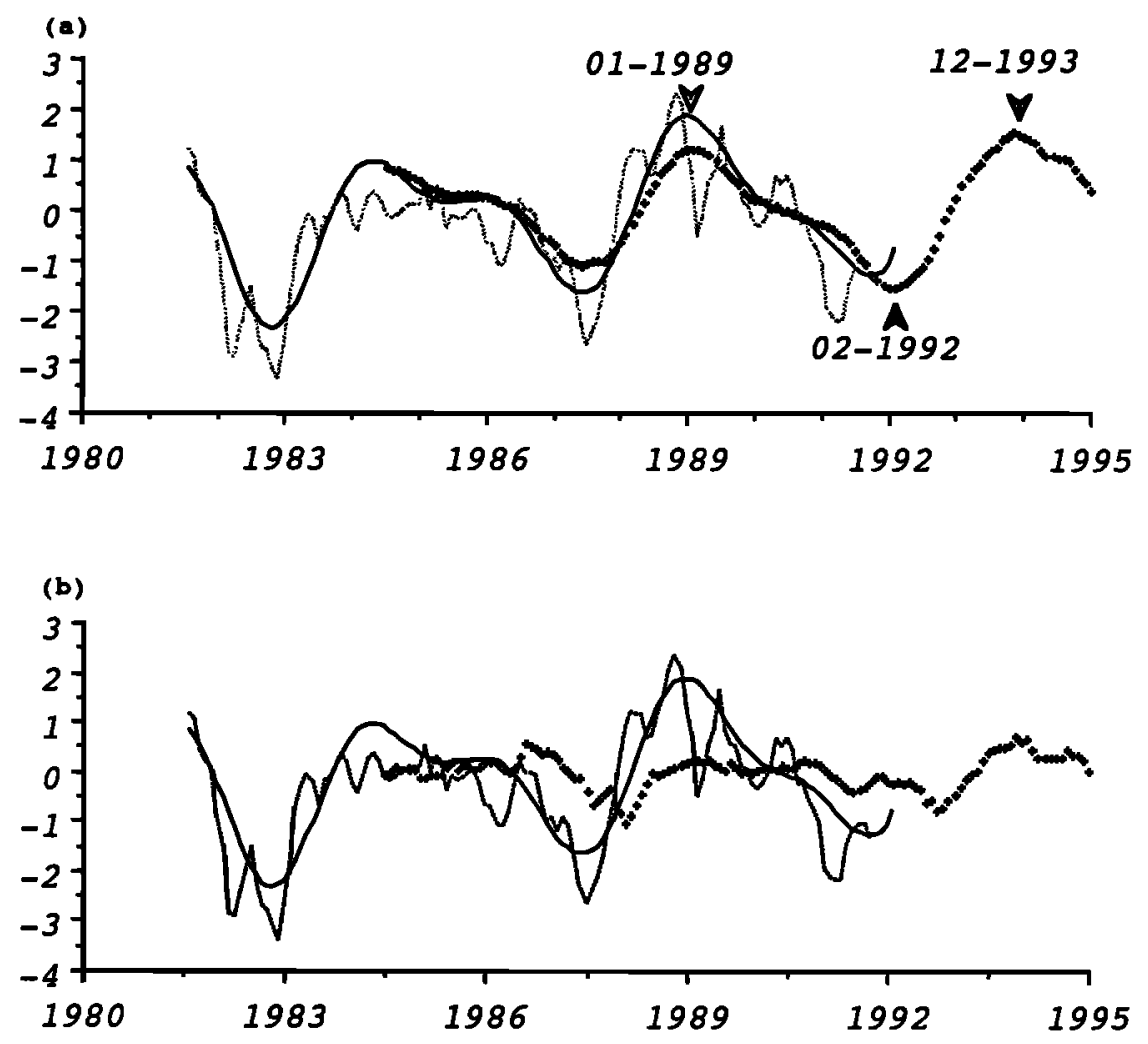

Fig. 4. Comparison of the last 10 years of the SSA-filtered (solid) and 5-month running mean (dotted) SOI of Figure 3, with the 36-month forecasts (crosses) obtained by (a) applying autoregression (AR) to the reconstructed components (RCs) which isolate the variability carried by each of T-PCs $1-4$, and combining the resulting forecasts and (b) by applying AR directly to the 5-month averaged SOI. The arrows point to the last El Niño and La Niña events and to the La Niña event forecasted by the present method for 1993-1994.

resulting T-EOFs $1-4$ and T-PCs $1-4$ are used to separate the variability associated with each of the four first SVs using Vautard et al.'s [1992] reconstruction formula (e'quation (2.17) there). MEM is applied to the four resulting RCs with a window width of 60 months [Burg, 1968; Penland et al., 1991].

The AR predictors so obtained are applied separately to the four prefiltered time series to issue a 36-month forecast for each of them. The corresponding ENSO forecast (leftmost cross in Figure $4 a$ ) is obtained by summing these four forecasts. One more raw SOI value is then added to the unfiltered data, and the T-PCs and corresponding prefiltered time series are recomputed. MEM is applied again to the latter and another 36-month forecast is made (second cross from the left in Figure $4 a$ ). The entire procedure is repeated 100 times to issue the 100 36-month ENSO forecasts for July 1984 to February 1995 shown in Figure $4 a$ (crosses). It is important to notice that no "look-ahead" is involved in the procedure, i.e., no information past the date from which a prediction is issued has been used, in either the hindcasting (validation dates prior to February 1992) or the forecasting (no validation available as yet) mode.

The Pearson product-moment correlation [Press et al., 1988] between the overlapping portions of the time series of 36-month forecasts (Figure $4 a$, crosses) and of the SSAfiltered SOI (Figure $4 a$, solid) is 0.966 . It is 0.772 for the hindcast of the 5-month running mean SOI (Figure $4 a$, dotted). The result of applying MEM directly to the 5-month running mean SOI is shown in Figure $4 b$, where the time series of 36-month forecasts so obtained (crosses) is compared to the SSA-filtered (solid) and 5-month running mean SOI (dotted). The Pearson correlations between these direct AR forecasts and the latter two time series, again over their overlapping portions, are 0.494 and 0.559 , respectively. The dramatic improvement in forecast skill obtained by applying the AR predictors to the four leading $\mathrm{RCs}$, rather than directly to the 5-month running mean SOI, stems from the SSA-filtered time series having simpler power spectra, and hence more robust low-order AR coefficients.

The first two arrows in Figure $4 a$ point to the extrema of the SSA-filtered SOI which correspond to the 1988-1989 LN and 1991-1992 EN events. The 5-month running mean SOI also peaked in January 1989 during the last LN. It is still too early at the time of writing to know whether its minimum corresponding to the current EN will also coincide with the minimum of the SSA-filtered SOI in February 1992. The SOI maximum in December 1993 (third arrow), if correctly predicted, could correspond to the next LN. This event could be associated with a drought over the continental United States during the second half of 1993, comparable to the 1988 drought, which has been plausibly associated with the 1988-1989 LN [Mo et al., 1991; Trenberth and Arkin, 1988]. Finally, an extension of the forecasts based on the entire data set through February 1992 also predicts an EN for 1996-1997 [Keppenne and Ghil, 1992], but the method's forecast skill at these long leads is considerably less than at 36 months.

At the time of writing, we have at our disposal the April 
1992 issue of the Climate Diagnostics Bulletin [Kousky, 1992]. The 5-month running mean SOI plotted in the bulletin is less smooth than the SOI used in this paper, making it difficult to identify a preferential trend, and the March observations [Kousky, p. 5] do not give a clear indication as to whether the 1991-1992 EN event has begun to weaken. The composite forecast from the statistical model of Barnett et al. [1988] and the dynamical model of Cane et al. [1986] show a cooling trend in the NINO3 sea surface temperature anomalies [Cane et al., 1986, p. 46], in agreement with our forecast.

\section{SUMMARY AND DiscuSSION}

The time series of the Southern Oscillation index (SOI) computed from the Darwin and Tahiti monthly mean sea level pressure records (Figure 1) was decomposed in terms of its temporal principal components and empirical orthogonal functions (T-PCs and T-EOFs) in order to separate the deterministic oscillations from noise [Vautard and Ghil, 1989]. The ENSO-related variability (Figure 3) corresponds to $21.7 \%$ of the total variance of the SOI. It can be separated into a 4- 6-year low-frequency component (Figure 2a), associated with T-PCs 1 and 2, and a 2- 3-year highfrequency component (Figure $2 b$ ), whose variance is carried by T-PCs 3 and 4 .

T-PCs $1-4$ are combined to provide a SSA-filtered SOI time series. This SOI is smoother than the 5-month averaged conventional SOI and can single out warm and cold events as well as the latter (Figure 3). Its smoothness provides it with the added advantage of being easier to forecast by a time series approach when the four reconstructed components (RCs), which isolate the variance associated with each of the four leading T-PCs, are forecasted individually (Figure 4). The autoregressive (AR) coefficients of the leading RCs are used for this purpose.

The SSA-filtered SOI has high predictability at leads of up to 36 months: the Pearson product-moment correlation between the 36-month forecasts and the validating time series is 0.966 , with no look-ahead involved. The application of the forecast method to the entire SOI data set, using data until February 1992, results in the prediction of a La Niña event for the 1993-1994 winter. The latter, if correctly predicted, could be associated with a late-1993 drought over the continental United States, comparable to the late-1988 drought which has been associated with the 1988-1989 La Niña [Mo et al., 1991; Trenberth and Arkin, 1988]. The results are compared with the latest observations and model forecasts published by the Climate Analysis Center [Kousky, 1992]. The two models, statistical [Barnett et al., 1988] and dynamical [Cane et al., 1986], agree with each other and with our AR results for the second half of 1992 .

Acknowledgments. It is a pleasure to thank Kingtse Mo for suggesting the use of SSA to predict SOI and for many pertinent references; Gene Rasmusson for sharing well before publication preliminary results of Rasmusson et al. [1990]; and Jean Dickey for her interest and encouragement. Stimulating discussions with Adam Freedman, Steve Marcus, Cécile Penland, and Robert Vautard were also very helpful. Two anonymous referees improved the presentation. This work is supported by the Caltech President's Fund, a grant from DOE's National Institute for Global Environmental Change (NIGEC) to UCLA, a grant from NOAA's Climate and Global Change program to UCLA and JPL, and a Guggenheim
Fellowship to Michael Ghil. M. G. would also like to thank the staff of the Laboratoire de Météorologie Dynamique du LNRS, Ecole Normale Supérieure, Paris, for their hospitality during his sabbatical.

\section{REFERENCES}

Akaike, H., A new look at the statistical model identification, IEEE Trans. Autom. Control, AC-19, 716-723, 1974.

Barnett, T. P., and R. W. Preisendorfer, Multifield analog prediction of short term climate fluctuations using a climate state vector, $J$. Atmos. Sci., 35, 1771-1787, 1978.

Barnett, T., N. Graham, M. Cane, S. Zebiak, S. Dolan, J. O'Brien, and D. Legler, On the prediction of the El Niño of 1986-87, Science, 24l, 192-196, 1988.

Broomhead, D. S., and G. P. King, Extracting qualitative dynamics from experimental data, Phys. D Amsterdam, 20, 217-236, 1986.

Burg, J. P., Maximum entropy spectral analysis, in Modern Spectrum Analysis, edited by D. G. Childers, pp. 34-48, IEEE Press, New York, 1968.

Bjerknes, J., Atmospheric teleconnections from the Equatorial Pacific, Mon. Weather Rev., 97, 163-172, 1969.

Cane, M., El Niño, Annu. Rev. Earth Planet. Sci., 14, 43-70, 1986.

Cane, M., S. Zebiak, and S. Dolan, Experimental forecasts of El Niño, Nature, 231, 827-832, 1986.

Childers, D. G. (Ed.), Modern Spectral Analysis, IEEE Press, New York, 1978.

Chu, P. S., and R. W. Katz, Modeling and forecasting the Southern Oscillation: a time-domain approach, Mon. Weather Rev., 113, 1876-1888, 1985.

Colebrook, J. M., Continuous plankton records: Zooplankton and environment, North-East Atlantic and North Sea, 1948-1975, Oceanol. Acta, 1, 9-23, 1978.

Deser, C., and J. M. Wallace, El Niño events and their relation to the Southern Oscillation: 1925-86, J. Geophys. Res., 92, 14,189$14,196,1987$.

Dickey, J. O., S. L. Marcus, and R. Hide, Global propagation of interannual fluctuations in atmospheric angular momentum, $\mathrm{Na}$ ture, 357, 484-488, 1992.

Fraedrich, K., Estimating the dimensions of weather and climate attractors, J. Atmos. Sci., 43, 419-432, 1986.

Fraedrich, K., El Niño/Southern Oscillation predictability, J. Atmos. Sci., 116, 1001-1002, 1988.

Ghil, M., and K. Mo, Intraseasonal oscillations in the global atmosphere, Part I, Northern Hemisphere and tropics, J. Atmos. Sci., 48, 752-779, 1991 a.

Ghil, M., and K. Mo, Intraseasonal oscillations in the global atmosphere, Part II, Southern Hemisphere, J. Atmos. Sci., 48, $780-790,1991 b$.

Ghil, M., and R. Vautard, Interdecadal oscillations and the warming trend in global temperature time series, Nature, 350, 324-327, 1991.

Ghil, M., M. Kimoto, and J. D. Neelin, Nonlinear dynamics and predictability in the atmospheric sciences, Rev. Geophys., 29, suppl. 46-55, 1991.

Graham, N. E., J. Michaelsen, and T. P. Barnett, An investigation of the El Niño-Southern Oscillation cycle with statistical models, 1, Predictor field characteristics, J. Geophys. Res., 92, 14,251$14,270,1987 a$.

Graham, N. E., J. Michaelsen, and T. P. Barnett, An investigation of the El Niño-Southern Oscillation cycle with statistical models, 2, Model results, J. Geophys. Res., 92, 14,271-14,289, 1987 b.

Grassberger, P., Do climatic attractors exist?, Nature, 323, 609-612, 1986.

Keppenne, C. L., and M. Ghil, Adaptive spectral analysis of the Southern Oscillation Index, in Proceedings of the XVth Annual Climate Diagnostics Workshop, pp. 30-35, U.S. Department of Commerce, NOAA, Springfield, Va., 1990.

Keppenne, C. L. and M. Ghil, Extreme weather events, Nature, $358,547,1992$.

Kousky, V. E. (Ed.), Climate Diagnostics Bulletin, 66 pp., Climate Analysis Center, Washington, D. C., 1992.

Lau, K. M., and P. H. Chan, Aspects of the 40-50 day oscillation during the northern summer as inferred from outgoing longwave radiation, Mon. Weather Rev., 114, 1354-1367, 1986.

Mañe, R., On the dimension of the compact invariant sets of certain 
nonlinear maps, in Dynamical Systems and Turbulence, edited by D. A. Rand and L. S. Young, pp. 230-242, Springer-Verlag, New York, 1981.

Mo, K. C., and M. Ghil, Interannual oscillations in the $700 \mathrm{mb}$ geopotential height field over the northern hemisphere, J. Clim., in press, 1992.

Mo, K. C., J. R. Zimmerman, E. Kalnay, and M. Kanamitsu, A GCM study of the 1988 United States drought, Mon. Weather Rev., 119, 1512-1532, 1991.

Neelin, J. D., et al., Tropical air-sea interaction in general circulation models, Clim. Dyn., 7, 73-104, 1992.

Penland, C., M. Ghil, and K. M. Weickmann, Adaptive filtering and maximum entropy spectra, with application to changes in atmospheric angular momentum, J. Geophys. Res., 96, 22,659-22,671, 1991.

Pike, E. R., J. G. McWhirter, M. Bertero, and C. de Mol, Generalized information theory for inverse problems in signal processing, IEE Proc., 131, 660-667, 1984.

Philander, S. G. H., El Niño, La Niña, and the Southern Oscillation, 293 pp., Academic, San Diego, Calif., 1990.

Preisendorfer, R. W., Principal Component Analysis in Meteorology and Oceanography, edited by C. D. Mobley, 425 pp., Elsevier, New York, 1988.

Press, W. H., B. P. Flannery, S. A. Teukolski, and W. T. Vetterling, Numerical Recipes in C, pp. 461-465, Cambridge University Press, New York, 1988.

Procaccia, I., Weather systems-Complex or just complicated?, Nature, 333, 498-499, 1988.

Rasmusson, E. M., and T. H. Carpenter, Variations in tropical sea surface temperature and surface wind fields associated with the Southern Oscillation/El Niño, Mon. Weather Rev., 110, 354-384, 1982.

Rasmusson, E. M., X. Wang, and C. F. Ropelewski, The biennial component of ENSO variability, J. Mar. Sys., I, 71-96, 1990.

Ruelle, D., Deterministic chaos-The science and the fiction, The Claude Bernard Lecture 1989, Proc. R. Soc. London, Ser. A, 427, 241-248, 1990.

Sarachik, E. S., Predictability of ENSO, in Ocean-Climate Interaction, edited by M. E. Schlesinger, pp. 161-171, Kluwer Academic, Boston, Mass., 1990.
Sauer, T., J. A. Yorke, and M. Casdagli, Embedology, J. Stat. Phys., 65(3-4), 579-616, 1991.

Schweigger, E., La "legitima" corriente del Nin̄o, Boln. Comp. Administr. Guano, 21, 255-296, 1945.

Takens, F., Detecting strange attractors in turbulence, in Dynamical Systems and Turbulence, edited by D. A. Rand and L. S. Young, pp. 366-381, Springer-Verlag, New York, 1981.

Trenberth, K. E., and P. A. Arkin, Origins of the 1988 NorthAmerican drought, Science, 242, 1640-1645, 1988.

Trenberth, K. E., and D. J. Shea, On the evolution of the Southern Oscillation, Mon. Weather Rev., 115, 3078-3096, 1987.

Vautard, R., and M. Ghil, Singular spectrum analysis in nonlinear dynamics, with applications to paleoclimatic time series, Phys. $D$ Amsterdam, 35, 395-424, 1989.

Vautard, R., P. Yiou, and M. Ghil, Singular spectrum analysis: A toolkit for short, noisy chaotic signals, Phys. D Amsterdam, 58, 95-126, 1992.

Walker, G., On periodicity in series of related terms, Proc. $R$. Soc. London, Ser. A, 131, 518-532, 1931.

Walker, G., and E. W. Bliss, World Weather IV, Mem. R. Meteorol. Soc., 4, 119-139, 1937.

Weare, B. C., and J. S. Nasstrom, Examples of extended empirical orthogonal function analyses, Mon. Weather Rev., 110, 481-485, 1982.

Whitney, H., Differentiable manifolds, Ann. Math., 37, 645-680, 1936.

Yule, G. U., On a method of investigating periodicities in disturbed series, Philos. Trans. R. Soc. London, Ser. A, 226, 267-298, 1927.

M. Ghil, Climate Dynamics Center and Department of Atmospheric Sciences and Institute of Geophysics and Planetary Physics, University of California, Los Angeles, CA 90024.

C. L. Keppenne, Jet Propulsion Laboratory, California Institute of Technology, Pasadena, CA 91109.

(Received October 1, 1991; revised July 6, 1992;

accepted September 10, 1992.) 